\title{
A Component-Level Model of Automatic Dependent Surveillance - Broadcast (ADS-B)
}

\author{
AIAA Aviation 2018 \\ June 25-29, 2018 \\ Michael M. Madden \\ NASA Langley Research Center \\ MS 125B \\ Hampton, VA 23681
}

\begin{abstract}
Automatic Dependent Surveillance - Broadcast (ADS-B) is being employed in numerous peer-to-peer initiatives attempting to expand the capacity of the National Airspace System (NAS) or enable mixed operations of manned and unmanned vehicles. Safety assessments of these initiatives rely, in part, on modeling the accuracy of ADS-B in reporting the position and direction of an ownship and surrounding traffic. Frequently, these initiatives utilize a position uncertainty model that applies a reported ADS-B estimation position uncertainty (EPU) value to a Rayleigh distribution and uses a Gauss-Markov random walk to add error to the ADS-B output of a vehicle. This model of ADS-B state error is easy to implement and apply to numerous problems. However, it has a couple of drawbacks. First, the ADS-B state errors are equally probable in all directions. This is a good assumption in situations where aircraft maneuvering is not constrained. However, in situations where the aircraft maneuvering is constrained such as landing, the error distribution is likely to exhibit directionality and the non-directional model may skew results especially when assessing very low probabilities (e.g., $10^{-9}$ ) of catastrophic encounters. Second, the model does not account for processing latency in the receiving aircraft. NASA Langley Research Center (LaRC) recently examined the feasibility of decreasing the spacing of aircraft on parallel approaches to runways separated by as little as 700 feet [Perry2013]. For Monte-Carlo analysis using a high-fidelity simulation of a large transport, LaRC started with a Gauss-Markov model of ADS-B error but then developed a component level model of ADS-B error to increase the fidelity of results.

This LaRC assessment of parallel approaches had a forward looking time frame of five to ten years. Therefore, the component level model assumes that the ADS-B system is fed directly from and synchronized with an autonomous Global Positioning System (GPS) receiver. This model covers the end-to-end reporting and consumption of ADS-B state from the GPS receiver on the transmitting aircraft to processing of the ADS-B report on the receiving aircraft. The model essentially divides the ADS-B path into four systems: the GPS receiver, the ADS-B OUT system, the ADS-B IN system, and the target application. A common error source in each system is latency and each system may vary in the duration of its processing latency and how much of that latency the system attempts to
\end{abstract}


compensate for. The model performs latency compensation for each system and the compensation error is the purely the difference between compensated state and the vehicle true state at the end of the latency duration. Additionally, at each interface, the state may lose precision due to the encoding used for the transmission. The reduced precision may increase error in downstream latency compensation. In summary, the error contributors for each system are outlined below:

- GPS Receiver

o Signal-in-space error for position and velocity modeled as a Gauss-Markov walking error.

o Processing latency including both compensated and uncompensated latency. However, the time of applicability reported for the data omits the uncompensated transmission latency.

o Precision reduction on output due to data encoding

- ADS-B OUT

o Processing latency including both compensated and uncompensated latency. ADS-B OUT also compensates for the uncompensated GPS transmission latency.

o Precision reduction on output due to data encoding

- ADS-B IN

o Processing latency including both compensated and uncompensated latency.

- $\quad$ Target Application

o Reception latency. In this study, the latency is assumed to be compensated.

This study models a WAAS GPS assuming it will be the minimum equipage for aircraft operating in five to ten years. RTCA DO-229D defines minimum operations standards for WAAS GPS receivers. However, the allowable position uncertainty of $32 \mathrm{~m}$ and update rate of $1 \mathrm{~Hz}$ best represent older generation models [RTCA2006]. RTCA DO-242, the MASPS for ADS-B, defines, in Appendix J, the expected $1 \sigma$ position error of WAAS GPS to be $1.8 \mathrm{~m}(2 \sigma=3.6 \mathrm{~m})$ in steady flight [RTCA2002]. Moreover, Garmin advertises WAAS GPS units for aviation with a $1 \mathrm{~m}$ RMS position error and $5 \mathrm{~Hz}$ update rate [Garmin2012]. For velocity, RTCA DO-260B, the MOPS for ADS-B OUT, identifies the expected 95\% velocity accuracy of GPS units to be $0.2 \mathrm{~m} / \mathrm{s}$ per axis (or $0.5 \mathrm{~m} / \mathrm{s}$ for ground speed magnitude assuming a Rayleigh distribution) in stable flight [RTCA2009]. For GPS unit latency, RTCA DO-242 Appendix K defines the expected latency from measurement to data transmission to be $0.3 \mathrm{~s}$ for high NACp applications [RTCA2002]. This latency is divided into $0.1 \mathrm{~s}$ for receiver operation and $0.2 \mathrm{~s}$ for state data transmission to destination. GPS units transmit the UTC time of applicability for the state data, which enables compensation of the transmission latency by the receiving unit; the time of applicability coincides with the time mark pulse emitted by the GPS unit, which is normally also the start of transmission. RTCA DO-260B implies that in high NACp applications, the ADS-B avionics must be synchronized on the time mark of the GPS unit and the GPS unit must be configured to emit time marks at a UTC sub-epoch (a 0.2 UTC subsecond) [RTCA2009]. Based on the information above, the GPS units were modeled with a $3 \mathrm{~m}$ position noise, a $0.5 \mathrm{~m} / \mathrm{s}$ ground-speed noise, a $5 \mathrm{~Hz}$ update rate on the UTC sub- 
epoch, and a 300-ms latency from truth state through transmission. In addition, the data transmitted has numerical precision as defined in RTCA DO-229D Appendix H: 8.38E$8^{\circ}$ for latitude and longitude, $0.125 \mathrm{ft}$. for altitude, $0.125 \mathrm{KT}$ for ground speed, and $0.0055^{\circ}$ for true track [RTCA2006].

For ADS-B OUT, the FAA rule for ADS-B permits a 2.0 s latency from state measurement to ADS-B OUT transmission; $0.6 \mathrm{~s}$ of the latency can be uncompensated [FAA2010]. These minimum requirements are compatible with the FAA minimum NACp of 8 (92.6 m) but not the study's minimum NACp of 10 (10 m). RTCA DO-242 Appendix K provides guidance for the end-to-end latency of ADS-B OUT in high NACp applications. The latency contribution from the GPS unit is as defined in the previous paragraph. The latency expected for the ADS-B avionics from reception of GPS data to transmission of the ADS-B report is $0.4 \mathrm{~s}$ [RTCA2002]. ADS-B units synchronized to the GPS time mark, as defined in DO-260B, should be able to apply compensation for the full latency and can assign a UTC sub-epoch as the time of applicability for the ADS-B OUT report. This will allow the receiving aircraft to further compensate for latencies downstream. ADS-B OUT reports also exhibit the precision defined in RTCA DO-260B. The encoding algorithm for position maintains an approximately $5 \mathrm{~m}$ precision in the North and East axes. Velocity precision is $1 \mathrm{KT}$ in the North and East axes [RTCA2009].

Latency for ADS-B IN was taken from RTCA DO-317A, the Minimum Operational Performance Standards for Aircraft Surveillance Applications. This RTCA document allocates $0.5 \mathrm{~s}$ to ADS-B IN latency [RTCA2011]. The ADS-B report does not further reduce the precision of the state data so no more precision truncation is performed. Target applications on aircraft normally run asynchronously to the ADS-B IN system, thus the worst case reception latency is equal to the execution step of the target application. Furthermore, the target application may not further compensate for latency; however, safety-critical applications should perform the compensation. In this study, the added latency of the target application is $200 \mathrm{~ms}$, all of it compensated. To simplify calculations, the total and compensated latencies of the target application are simply added to the ADS-B IN latency since no data precision truncations are assumed to occur between the ADS-B IN system and the target application.

Because the state data is closely associated with a UTC sub-epoch in both the transmitter and the receiver, nearly all the latency can be compensated. However, there are some allowances to decrease the compensated latency. RTCA DO-260B allows up to a 5-ms deviation in the GPS time mark from the actual UTC sub-epoch [RTCA2009]. This deviation plus an additional $1 \mathrm{~ms}$ of uncaptured time delays is subtracted from the compensated latency in both the ADS-B OUT and ADS-B IN systems. The 5-ms GPS time mark deviation is also used to establish a worst-case clock difference between aircraft of $10 \mathrm{~ms}$ to which an additional $1 \mathrm{~ms}$ is added for other uncaptured time delays. This $11 \mathrm{~ms}$ uncompensated latency is assigned to the ADS-B IN processing of the traffic data. The resulting end-to-end latency of aircraft in this study is summarized below. Moreover, the summary provides the latency for two different paths. An ownship path that traverses locally from GPS to target application and a traffic path that traverses from the GPS of the traffic vehicle to the target application on the ownship (i.e. GPS $\rightarrow$ ADS-B OUT $\rightarrow$ 
ADS-B IN $\rightarrow$ Target Application). In the traffic path, the latency of the traffic data is divided between ADS-B OUT and ADS-B IN because of the change in precision of the state data in the ADS-B OUT report.

- Ownship Path

o Total Latency (GPS $\rightarrow$ Target Application): $500 \mathrm{~ms}$

o Compensated Latency: $494 \mathrm{~ms}$

- Traffic Path

o ADS-B OUT

- Total Latency (GPS $\rightarrow$ ADS-B OUT): $700 \mathrm{~ms}$

- Compensated Latency: 694 ms

o ADS-B IN

- Total Latency (ADS-B IN $\rightarrow$ Target Application): $700 \mathrm{~ms}$

- Compensated Latency: 689 ms

This paper will then present the error profiles of this component based model and compare it to a Gauss-Markov model for both a landing scenario and a path following scenario with turns.

\section{References}

[FAA2010] FAA. "Automatic Dependent Surveillance - Broadcast (ADS-B) Out Performance Requirements to Support Air Traffic Control (ATC) Service; Final Rule." Federal Register (National Archive and Records Administration) 75, no. 103 (May 2010): $30160-30195$.

[Garmin2012] Garmin. "Datasheet for GPS 500W, GPS 400W, and GNC 420.” https://buy.garmin.com/shop/store/assets/pdfs/specs/gps400w_spec.pdf (accessed September 4, 2012).

[Perry2013] Perry, Raleigh B.; Michael M. Madden, Wilfredo Torres-Pomales, and Ricky W. Butler: The Simplified Aircraft-based Paired Approach with the ALAS Alerting Algorithm, NASA/TM-2013-217804.

[RTCA2002] RTCA, Inc: "Minimum Aviation System Performance Standards for Automatic Dependent Surveillance Broadcast (ADS-B)”, DO-242A, Washington, D.C., June 25, 2002.

[RTCA2006] RTCA, Inc: "Minimum Operational Performance Standards for Global Positioning System / Wide Area Augmentation System Airborne Equipment”, DO229D, Washington, D.C., December 13, 2006.

[RTCA2009] RTCA, Inc: "Minimum Operational Performance Standards for $1090 \mathrm{MHz}$ Extended Squitter Automatic Dependent Surveillance - Broadcast (ADS-B) and Traffic Information Services - Broadcast (TIS-B)”, DO-260B, Washington, D.C., December 2, 2009. 
[RTCA2011] RTCA, Inc: "Minimum Operational Performance Standards (MOPS) for Aircraft Surveillance Applications (ASA) System”, DO-317A, Washington, D.C., December 13, 2011. 